\title{
Pratiques
}

Linguistique, littérature, didactique

169-170 | 2016

Enseignement/apprentissage de la langue, des textes et des discours. 40\&nbspans de Pratiques

\section{Les expansions du nom, côté élèves et côté manuels : représentations et pratiques scolaires}

A Pedagogical Perspective on Noun Modification

\section{Céline Corteel}

\section{OpenEdition}

Journals

Édition électronique

URL : http://journals.openedition.org/pratiques/3071

DOI : $10.4000 /$ pratiques.3071

ISSN : 2425-2042

Éditeur

Centre de recherche sur les médiations (CREM)

Référence électronique

Céline Corteel, «Les expansions du nom, côté élèves et côté manuels : représentations et pratiques scolaires », Pratiques [En ligne], 169-170 | 2016, mis en ligne le 30 juin 2016, consulté le 19 avril 2019 URL : http://journals.openedition.org/pratiques/3071; DOI : 10.4000/pratiques.3071

Ce document a été généré automatiquement le 19 avril 2019

(c) Tous droits réservés 


\title{
Les expansions du nom, côté élèves et côté manuels : représentations et pratiques scolaires
}

\author{
A Pedagogical Perspective on Noun Modification
}

\section{Céline Corteel}

\section{Introduction}

1 Notre contribution portera sur les modificateurs du nom dans le cadre du GN étendu, désignés sous le terme $d^{\prime}$ « expansions du nom ${ }^{1}$, et envisagés du double point de vue des élèves et de la littérature scolaire. Il ne s'agit donc pas d'interroger linguistiquement la notion, pour laquelle nous adoptons la définition proposée dans la grammaire de référence de M. Riegel et al. (1994) ${ }^{2}$, mais de l'aborder selon une perspective didactique, l'objectif étant de sonder les représentations et compétences des élèves pour pouvoir ensuite les confronter au traitement des expansions dans les supports d'apprentissage que constituent les manuels.

2 Pour cela, nous commencerons par présenter le principe et les résultats d'une enquête que nous avons menée auprès d'élèves de début de collège, puis nous dresserons un état des lieux du traitement des expansions du nom dans les manuels, en nous intéressant d'une part aux dispositifs didactiques mis en place pour aborder la notion, mais aussi à la description qui en est faite dans les leçons, ce qui permettra au passage de mesurer l'écart éventuel entre le discours scolaire et les grammaires de référence.

\section{Du côté des élèves}

3 Notre démarche a consisté en l'élaboration d'un questionnaire, qui a ensuite été soumis à 120 élèves issus de quatre classes de sixième différentes ${ }^{3}$. 


\subsection{Public sondé et principe du questionnaire}

4 Notre choix d'interroger des élèves de début de collège se fonde sur le fait que la notion d'expansion du nom est censée avoir été abordée au primaire, les programmes de 2008 en vigueur au moment de la réalisation de notre sondage stipulant que les élèves doivent savoir procéder à «l'identification des éléments du groupe nominal et de leurs fonctions $^{4}$ : déterminant, adjectif qualificatif épithète, complément du nom, proposition relative complément du nom ».

5 Le questionnaire proposé, reproduit dans son intégralité en annexe, se décompose en quatre activités. Nous avons d'abord demandé aux élèves d'expliquer avec leurs propres mots ce qu'est une expansion du nom, en précisant à quoi ça sert et en donnant des exemples. Les trois autres exercices, sur lesquels nous allons revenir dans la section 1.2., étaient tous constitués d'un corpus de phrases, précédé d'une consigne portant sur des constituants ciblés. Venons-en maintenant à l'analyse des réponses proposées par les élèves.

\subsection{Ce que suggèrent les réponses des élèves}

Un examen attentif des réponses fournies permet de mettre en lumière plusieurs grandes tendances, qui se dégagent au fil des différentes activités proposées.

\subsubsection{Activité 1 : « Définir une expansion »}

7 Pour ce qui est de la définition des expansions, d'abord, la grande majorité des élèves appréhende la notion sous un angle sémantique, avec un recours massif aux notions de « détail » et de «précision » sur le nom. On observe que $85 \%$ des exemples spontanés mettent un jeu un adjectif qualificatif, lequel figure parfois indûment en position attribut (cf. "Les cerises sont moisies et noires »). Sur le plan syntaxique, aucun élève ne mentionne explicitement le fait que la modification se joue dans le cadre du groupe nominal. Seul un tiers des sondés évoque la classe grammaticale des expansions, et les réponses fournies sont alors plutôt lacunaires qu'erronées. Les deux grandes tendances tiennent à la mention systématique de l'adjectif qualificatif, et au fait que le groupe prépositionnel n'est, à l'inverse, jamais mentionné (on dénombre toutefois 5 mentions du « complément du nom »). Enfin le caractère facultatif des expansions n'est signalé que dans $10 \%$ des cas, avec des justifications du type «On peut s'en passer sans changer le sens de la phrase ».

De façon intéressante, on constate aussi que $20 \%$ des élèves n'ont pas fait l'activité 1 , alors même qu'ils ont répondu aux autres questions. Ce taux d'abstention illustre vraisemblablement la difficulté des élèves à adopter une posture réflexive de type métalinguistique vis-à-vis des faits de langue grammaticaux.

\subsubsection{Activité 2 : « Identifier la nature et la fonction d'une expansion »}

9 La consigne de l'activité 2 demande aux élèves d'indiquer la nature et la fonction de constituants donnés, sans qu'il soit précisé qu'il s'agit chaque fois d'une expansion du nom. Le corpus proposé, volontairement constitué de phrases plutôt que de GN isolés, est le suivant : 
10 1. Cette pièce est remplie d'objets anciens et de meubles qui sentent la cire. 2. La danseuse porte une magnifique robe à paillettes. 3. Jules a acheté un ordinateur d'un prix excessif. 4. Cet homme a commis un crime que l'on ne peut pas accepter.

11 Signalons d'abord que beaucoup d'élèves qui proposent des éléments de définition pertinents dans l'activité 1 produisent ici des réponses complètement farfelues, ce qui suggère l'existence d'un fossé entre la capacité de restitution d'une leçon, et la capacité d'analyse de la langue en contexte.

12 Concernant l'adjectif qualificatif de la phrase 1, il a été massivement identifié (80\% des réponses), mais sa fonction d'épithète n'est en revanche mentionnée que dans $3 \%$ des cas, l'erreur la plus fréquente consistant à l'assimiler à un COD.

13 Pour ce qui est des GN prépositionnels proposés en 2 et 3 , un seul élève mentionne la présence de la préposition, les autres réponses les assimilant essentiellement à un nom (dans le cas de « une robe à paillettes») ou à un groupe nominal (dans le cas de «un ordinateur d'un prix excessif »). La fonction complément du nom, citée dans seulement $5 \%$ des cas, est quant à elle le plus souvent assimilée à un COI (« d'un prix excessif » étant parfois analysé comme le «COI de ordinateur»), ou comme un "complément circonstanciel de manière " dans le cas de "une robe à paillettes " (ces dernières réponses se basant vraisemblablement sur un raisonnement du type : « une robe comment ? - à paillettes »). Au-delà du caractère strictement incorrect de ces réponses, un point essentiel tient au fait qu'elles font état d'intuitions tout à fait pertinentes sur la langue, à savoir la conscience d'un support nominal pour les analyses du type " COI de ordinateur ", ou du caractère facultatif des expansions dans le cas où elles sont assimilées à des circonstants.

14 La relative de la phrase 4, enfin, a été identifiée comme une subordonnée dans un tiers des cas, le plus souvent « conjonctive $»^{5}$.

\subsubsection{Activités 3 et $4:$ « Ajout et repérage d'expansions »}

15 Dans l'activité 3, dont le corpus est reproduit ci-dessous, les élèves devaient réécrire des phrases en rajoutant des expansions à certains noms donnés :

16 1. Mes parents ont acheté un chien. 2. Cette femme ressemble beaucoup à son père. 3. Mes voisins vendent leur maison.

17 Outre le recours massif aux adjectifs épithètes, on observe ici que beaucoup élèves produisent des circonstants, avec des propositions du type Mes voisins vendent leur maison parce qu'ils déménagent.

18 Dans l'activité 4 , où il s'agit d' « entourer les expansions des noms en gras " ${ }^{6}$, on observe que la relative est le plus souvent omise, ce qui peut être mis au compte du fait qu'elle est rarement abordée en même temps que les autres expansions au primaire (pour ce qui est des manuels, du moins, cf. section 2 infra $)^{7}$. La deuxième grande tendance vient de ce que certains constituants, notamment le déterminant du nom expansé et l'adjectif attribut, sont indûment assimilés à des expansions. En particulier, dans l'énoncé attributif «Le frère de mon voisin est malade ", beaucoup d'élèves identifient " malade " comme une expansion de « frère ». On peut y voir la preuve de l'intuition d'un support nominal pour l'adjectif, mais aussi peut-être une conséquence des approches exclusivement sémantiques du rôle des mots, qui en passant à côté du caractère [+/- facultatif] des constituants permettent la confusion entre les fonctions attribut et épithète. 


\subsection{Constats d'ensemble et pistes didactiques pour la grammaire de phrase}

19 Au-delà de la spécificité des différentes activités proposées, quelques grands constats se dégagent au terme de ce petit sondage côté élèves. Le premier d'entre eux tient au caractère manifestement problématique du métalangage, avec le recours à une terminologie le plus souvent inadaptée ou même inventée, aboutissant alors à un jargon grammatical vide de sens. Or si «nommer n'est pas comprendre » comme le souligne fort justement C. Tisset (2005, p. 13), il n'en reste pas moins que la maitrise du métalangage est une condition nécessaire à une réflexion éclairée sur la langue.

L'autre constat, partiellement lié, concerne la confusion fréquente entre la nature et la fonction d'un constituant donné ${ }^{8}$, ce qui suggère que les élèves manient des concepts sans avoir forcément de vision d'ensemble des différents plan de l'analyse grammaticale d'une part, ni des différentes rôles possibles des constituants dans la phrase d'autre part.

Concernant spécifiquement les fonctions, outre le fait qu'elles sont largement méconnues, on observe qu'elles sont très rarement énoncées en termes relationnels, et que la distinction entre un complément essentiel et un adjoint non essentiel n'a souvent rien d'évident. À cet égard, les propositions de Delhay (2014), qui plaide en faveur d'une simplification terminologique basée sur une grammaire des constructions syntaxiques, semblent tout à fait pertinentes.

Enfin, si les difficultés d'analyse sont réelles, tout n'est pas négatif cependant, puisqu'ainsi que nous l'avons montré, les réponses fournies par les élèves témoignent aussi d'intuitions justes sur la langue, qui constituent autant de leviers potentiels pour la classe. L'assimilation du complément du nom à un COI, par exemple, suggère un repérage de la préposition, même si cette dernière n'est par ailleurs jamais mentionnée. Citons aussi le fait que la confusion avec les circonstants peut s'expliquer par la conscience du caractère facultatif du constituant.

Mais venons-en maintenant à la seconde partie de notre contribution, qui porte sur l'examen de la littérature scolaire en matière d'expansions.

\section{Du côté des manuels}

Du côté des ouvrages scolaires, notre corpus de travail se constitue de 12 manuels de $\mathrm{CM} 2$ postérieurs à 2008 , se réclamant tous des programmes en vigueur au moment de l'élaboration du présent travail.

\subsection{Corpus de travail}

25 La liste ci-dessous, basée sur le classement alphabétique des titres d'ouvrages ${ }^{9}$, recense l'ensemble des 12 manuels de notre corpus :

1. Au rythme des mots - Étude de la langue, Bordas (2011)

2. Français - Étude de la langue, CM1-CM2, Hatier (2011)

3. Français livre unique - Collection Caribou, Istra (2010)

4. Interlignes - Étude de la langue, Éditions Sed (2010) 
5. L'île aux mots, Nathan (2009)

6. Les nouveaux outils pour le français, Magnard (2013)

7. Mille-feuilles, Nathan (2013)

8. Mot de Passe, Hachette Éducation (2011)

9. Mots d'école - Mon livre de français, Éditions Sed (2012)

10. Mots en herbe, Bordas (2013)

11. Par mots et par phrases - Étude de la langue, Cycle 3, Nathan (2012)

12. Pépites Français CM2, Magnard (2013)

Dans ce qui suit, nous procéderons en trois temps. Après un rapide examen de la place dédiée aux expansions dans la partie grammaire des manuels, nous nous pencherons sur les détails du dispositif didactique associé à la notion d'expansion du nom, en analysant d'abord les modalités d'entrée dans la notion, puis la leçon proposée. Par commodité, le renvoi aux manuels se fera chaque fois à l'aide des numéros de la présente liste.

\subsection{Les expansions du nom dans les sommaires des manuels}

Notre analyse du traitement des expansions dans la partie " grammaire » des sommaires se fonde sur le titre et la place des différents chapitres dédiés à la notion.

\subsubsection{Données recueillies}

Le tableau suivant recense les données recueillies pour notre analyse :

Tableau 1. Critères d'analyse de la place dédiée aux expansions dans la partie grammaire des manuels

\begin{tabular}{|c|c|c|c|}
\hline $\begin{array}{l}\mathrm{N}^{\circ} \mathrm{du} \\
\text { manuel }\end{array}$ & $\begin{array}{l}\text { Intitulé des } \\
\text { rubriques de la } \\
\text { partie grammaire }\end{array}$ & $\begin{array}{l}\text { Titre du chapitre }{ }^{10} \\
\text { d'approche globale } \\
\text { des expansions }\end{array}$ & $\begin{array}{l}\text { Chapitres complémentaires, } \\
\text { dédiés spécifiquement à une ou } \\
\text { plusieurs expansion(s) }\end{array}$ \\
\hline 1 & $\varnothing$ & $\begin{array}{l}\text { «10. Le groupe } \\
\text { nominal » (p. } 41)\end{array}$ & $\begin{array}{l}\text { «15. L'adjectif qualificatif» (p. 59); «18. } \\
\text { La proposition subordonnée relative " } \\
(\text { p. 67); «19. Le complément du nom » } \\
(\text { p. 69) }\end{array}$ \\
\hline 2 & $\begin{array}{l}\text { La phrase (1); } \\
\text { Autour du verbe; } \\
\text { Autour du nom; La } \\
\text { phrase (2) }\end{array}$ & $\varnothing$ & $\begin{array}{l}\text { "Autour du nom: } 11 . \quad \text { L'adjectif } \\
\text { qualificatif (p. 30); 13. Le complément du } \\
\text { nom (p. 34); 14. La proposition relative } \\
\text { (p. 36)» }\end{array}$ \\
\hline 3 & $\varnothing$ & $\begin{array}{l}\text { «6. Le groupe } \\
\text { nominal } »(p .46)\end{array}$ & $\begin{array}{l}\text { «9. L'adjectif qualificatif (p. 60)»; «10. } \\
\text { Le complément du nom (p. } 74) ; \text { «20. La } \\
\text { proposition subordonnée relative } \\
\text { (p. 142)» }\end{array}$ \\
\hline 4 & $\varnothing$ & $\begin{array}{l}\text { «2. Les expansions } \\
\text { du nom } »(p .17)\end{array}$ & «15. La proposition relative » (p.56) \\
\hline
\end{tabular}




\begin{tabular}{|c|c|c|c|}
\hline 5 & $\varnothing$ & $\begin{array}{l}\text { «11. Compléter un } \\
\text { nom » (p. 199) }\end{array}$ & $\begin{array}{l}\text { «12. Identifier la proposition } \\
\text { subordonnée relative }(\text { (p. 201) }\end{array}$ \\
\hline 6 & $\begin{array}{l}\text { La phrase et le texte; } \\
\text { Autour du verbe; } \\
\text { Autour du nom }\end{array}$ & $\begin{array}{lr}\text { «Autour du } & \text { nom }[\ldots]: \\
1 . \quad \text { le } & \text { groupe } \\
\text { nominal } » & (\text { p. } 40)\end{array}$ & $\begin{array}{l}\text { «La phrase et le texte: 9. La phrase } \\
\text { complexe: la proposition relative» (p. } \\
24) ; \\
\text { «Autour du nom : } 3 \text {. L'adjectif qualificatif } \\
\text { (p. 46); 5. Le complément du nom et la } \\
\text { proposition relative» (p. 50) }\end{array}$ \\
\hline 7 & $\begin{array}{l}\text { La phrase; Les } \\
\text { classes de mots (ou la } \\
\text { nature des mots); } \\
\text { Les fonctions }\end{array}$ & $\begin{array}{l}\text { «Les fonctions }[\ldots]: \\
18 . \text { Les expansions } \\
\text { du groupe nominal » } \\
(p .180)\end{array}$ & \\
\hline 8 & $\varnothing$ & 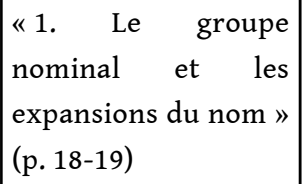 & $\begin{array}{l}\text { « 4. L'adjectif qualificatif (épithète ou } \\
\text { attribut)» (p. 60); «6. Le complément du } \\
\text { nom (avec la proposition relative) » (p. } \\
90)\end{array}$ \\
\hline 9 & $\varnothing$ & $\begin{array}{l}\text { «4. Connaître les } \\
\text { différents éléments } \\
\text { du groupe nominal » } \\
(\text { p. } 46)\end{array}$ & $\begin{array}{l}\text { «5. Accorder l'adjectif qualificatif et } \\
\text { identifier ses fonctions » (p. 69); «8. } \\
\text { Reconnaître le complément du nom » (p. } \\
92) \text {; «19. Reconnaître la proposition } \\
\text { relative » (p. 227) }\end{array}$ \\
\hline 10 & $\varnothing$ & $\begin{array}{l}\text { « } 5 . \quad \text { Le groupe } \\
\text { nominal } »(p .172)\end{array}$ & $\begin{array}{l}\text { «14. La proposition relative »(p. 190); } \\
\text { «15. Le complément du nom »(p. 192) }\end{array}$ \\
\hline 11 & $\begin{array}{l}\text { Autour du verbe; } \\
\text { Autour du nom; } \\
\text { Autour de la phrase; } \\
\text { Autour du texte }\end{array}$ & $\varnothing$ & $\begin{array}{l}\text { «Autour du nom: 10. L'adjectif } \\
\text { qualificatif» (p. 30); «11. Le complément } \\
\text { du nom » (p. 32); «12. La proposition } \\
\text { relative » (p. } 34) ; \\
\text { «Autour de la phrase : 23. Les propositions } \\
\text { subordonnées relatives» (p. 58) }\end{array}$ \\
\hline 12 & $\begin{array}{l}\text { La phrase; Les } \\
\text { classes de mots; Les } \\
\text { fonctions }\end{array}$ & $\begin{array}{l}\text { «es fonctions }[\ldots]: \\
19 . \quad \text { Identifier les } \\
\text { expansions du nom » } \\
(\text { p. 150) }\end{array}$ & $\begin{array}{l}\text { «La phrase : 4. Identifier la proposition } \\
\text { subordonnée relative» (p. 120); } \\
\text { « Les fonctions : 18. Identifier les fonctions } \\
\text { de l'adjectif » (p. 148) }\end{array}$ \\
\hline
\end{tabular}

Avant d'examiner ce que ces données nous apprennent de la place dévolue aux expansions (section 2.2.3), arrêterons-nous un instant sur le cadre grammatical de référence des manuels.

\subsection{2. Écoles grammaticales de référence}

Le caractère composite de la grammaire scolaire, étudié notamment par C. Vargas (2004 ; 2009), est manifeste dans notre corpus, dans la mesure où plusieurs écoles grammaticales sous-tendent implicitement les sommaires de la rubrique "grammaire». Cela ressort 
clairement des intitulés et de la progression des différents chapitres, qui empruntent essentiellement à trois cadres d'analyse implicites: celui de l'analyse logique traditionnelle, celui de la grammaire distributionnelle héritière du structuralisme, tout comme celui plus récent de la grammaire de texte inspirée de la linguistique textuelle.

31 Le manuel 1, par exemple, qui consacre les cinq premiers chapitres de grammaire aux notions de phrase («verbale» ou «non verbale»; «simple» ou « complexe») et de proposition («indépendante» vs coordination et juxtaposition; «principale» ou «subordonnée ») semble emprunter les voies de l'approche logique traditionnelle. La notion d'expansion du $\mathrm{N}$ y est abordée dans le chapitre 10. De façon inverse, la progression du manuel 4 relève d'une logique plus distributionnelle: les 11 premiers chapitres portent sur les notions de "natures et fonctions ", la phrase et la proposition étant quant à elles abordées dans les 4 derniers chapitres. Dans ce manuel, les expansions du nom sont abordées dès le chapitre 2. Par ailleurs, certains sommaires incluent également une approche textuelle de la grammaire, comme le manuel 11 qui consacre, dans une rubrique intitulée « Autour du texte », 2 chapitres aux « substituts du nom » et 1 chapitre à la « cohérence temporelle ».

32 À noter aussi que la logique d'ensemble qui préside à l'organisation des différents chapitres de grammaire reste le plus souvent implicite. Dans 7 manuels du corpus, la rubrique grammaire se présente ainsi sous la forme d'une succession de notions sans lien explicite. En outre les livrets d'accompagnement du maitre insistent davantage sur la possibilité d'une approche souple, choisie par le maitre en fonction de sa progression, que sur ce qui a motivé la progression du manuel. À cet égard, le manuel 5 se démarque toutefois des autres dans la mesure où le guide pédagogique associé spécifie: «En grammaire, on distingue deux ensembles: Unités 2 à 8 : la phrase et ses constituants (le groupe sujet, le groupe verbal avec les différents compléments); Unités 9 à 13: les composants du groupe nominal et ses substituts ».

33 Dans les sommaires qui subdivisent la grammaire en sous-parties distinctes, deux logiques différentes semblent à l'œuvre. Deux d'entre eux (manuels 7 et 12) proposent une approche basée sur la distinction nature/fonction. Dans les 3 autres cas (manuels 2, 6 et 11) l'entrée se fait en revanche par les parties du discours, et une rubrique intitulée "Autour du verbe », en précède une autre intitulée " Autour du nom ». À cela s'ajoute la présence d'une ou plusieurs rubrique(s) dévolue(s) à la phrase et au texte, placée(s) selon les cas en début et/ou en fin de sommaire. Cette organisation semble faire écho au rapport d'A. Bentolila (2006) sur l'enseignement de la grammaire au cycle 3, qui plaidait pour une distinction explicite en classe entre les compléments du verbe, de la phrase, et du nom (cf. ibid., p. 20-21, 29) ${ }^{11}$.

34 Au-delà de ces grandes tendances, l'ordre des différents chapitres semble parfois aléatoire, et il n'est pas toujours évident de saisir le principe qui préside à l'organisation de la rubrique grammaire ${ }^{12}$, même si la tendance générale est à l'alternance plus ou moins régulière de chapitres dédiés à l'approche d'une fonction, et d'autres consacrés à l'approche d'une classe grammaticale donnée. Les données examinées confirment ainsi l'analyse de C. Vargas (2009, p. 29), pour qui « la grammaire scolaire n'est pas obtenue pas transposition didactique de savoirs savants, mais par recomposition de savoirs hétérogènes, certains obtenus à partir de la linguistique par emprunts-modifications, d'autres conservés de la grammaire scolaire déjà-là. Par recomposition, nous entendons que ces savoirs sont issus d'horizons très différents, ne sont pas véritablement articulés, 
synthétisés, mais sont juxtaposés, alors même qu'ils peuvent être inconciliables entre eux. »

\subsubsection{Place dédiée aux expansions} sommaires. On observe qu'elle fait l'objet d'un chapitre explicitement dédié dans 4 manuels $\left(n^{\circ} 4,5,7,12\right)$. Tous recourent à la terminologie «d'expansions » dès le titre ${ }^{13}$, à l'exception du manuel 5 qui comporte un chapitre intitulé « Compléter un nom ». Dans 6 autres manuels $\left(n^{\circ} 1,3,6,8,9,10\right)$, la notion est abordée dans un chapitre portant plus largement sur l'ensemble du GN. Seul le manuel 8 recourt au terme d'expansion dans le titre (cf. «Le groupe nominal et les expansions du nom»). Les autres s'intitulent «le groupe nominal » (manuels 1, 3, 6, 10) ou « Connaître les différents éléments du groupe nominal » (manuel 9). En consultant les chapitres en question, on retrouve chaque fois la terminologie d'« expansions du nom ». Enfin, 2 manuels se démarquent des autres en ne proposant pas de chapitre global sur les expansions. Cette démarcation reste toutefois relative dans la mesure où l'un (manuel 2) renvoie les élèves à un mémo, lequel se présente sous la forme d'un petit livret distinct du manuel et consacre une fiche entière aux « expansions du nom ». Le manuel 11 récapitule quant à lui la liste des différentes expansions dans la synthèse finale de la rubrique dédiée au nom. D'un point de vue strictement terminologique, enfin, le recours au terme d'« expansion » se retrouve dans l'ensemble des manuels, à l'exception des manuels 1 et 5 .

L'hétérogénéité du corpus tient aussi à une certaine disparité dans le traitement des différentes expansions. Si dans tous les cas (à l'exception du manuel 7), une ou plusieurs expansions sont ensuite abordées séparément dans des chapitres distincts, les approches diffèrent sensiblement ${ }^{14}$ :

- 5 manuels $\left(n^{\circ} 1,2,3,9,11\right)$ proposent un chapitre spécifique pour chaque type d'expansion ( " adjectif », « complément du nom », « relative »);

- 2 manuels ( $n^{\circ} 6$ et 8 ) consacrent un chapitre à « l'adjectif qualificatif » et abordent ensuite ensemble « le complément du nom et la relative»;

- 1 manuel $\left(n^{\circ} 10\right)$ dédie un chapitre spécifique à la «proposition relative » et un autre au « complément du nom »;

- 1 manuel $\left(n^{\circ} 12\right)$ consacre un chapitre spécifique à la relative, et un à l'adjectif ;

- 2 manuels $\left(n^{\circ} 4,5\right)$ dédient un chapitre spécifique à la seule relative.

Un point commun tient ainsi au statut particulier de la relative, qui est la seule à faire systématiquement l'objet d'un chapitre dédié ${ }^{15}$. Deux manuels ( ${ }^{\circ} 6$ et 11 ) lui consacrent même 2 chapitres: l'un dans la rubrique "Autour du nom» (selon une approche d'influence structuraliste), l'autre dans la rubrique sur la phrase (conformément à l'approche logique traditionnelle). Autrement dit, le phénomène d'influences plurielles, voire contradicatoires (cf. sur ce point C. Vargas, 2009, p. 30-32) mentionné supra, s'observe parfois à l'échelle d'un seul et même manuel.

\subsubsection{Ce que suggèrent les sommaires : premier bilan}

Ce premier tour d'horizon montre d'abord que la notion d'expansion du nom ne jouit pas d'une visibilité systématique dans le paysage éditorial (contrairement à d'autres 
phénomènes mieux circonscrits, comme par exemple l'accord du verbe avec son sujet dans le domaine orthographique). Le plus souvent, la notion est mentionnée dans le chapitre sur le GN, et les différentes expansions font ensuite l'objet de chapitres distincts, parfois fort éloignés. Dans le manuel 3, l'adjectif épithète est ainsi abordé en début d'ouvrage juste après le nom (p. 60), tandis que la relative arrive en fin de progression, après des considérations sur la phrase complexe (p. 142). Ce statut « à part » de la relative constitue d'ailleurs une constante, alors même que l'on peut s'interroger sur le bienfondé d'un tel choix qui propose finalement une approche morcelée de constituants jouant le même rôle dans le GN. La position de J. Gardes-Tamine (2005, p. 139) sur ce point est tranchée : selon elle, « c'est dans les expansions, avec les adjectifs, qu'il faut aborder la proposition relative, et non dans un chapitre au demeurant discutable sur les subordonnées, car à la différence des complétives, elle ne constitue pas un SN, mais a un fonctionnement adjectival».

\subsection{L'entrée dans la notion}

40 Venons-en maintenant plus spécifiquement à la façon dont la notion d'expansion est abordée dans les séances dédiées des manuels.

\subsubsection{Démarche d'ensemble}

41 Dans tous les ouvrages de notre corpus, la démarche adoptée se veut inductive, puisque la leçon n'est jamais donnée d'emblée, mais arrive après une phase d'observation au cours de laquelle l'élève est censé la construire lui-même.

42 À deux exceptions près, dont nous dirons un mot infra, l'entrée dans la notion se fait à partir d'un texte support, de nature documentaire ou extrait de la littérature jeunesse. Sur la principe, la démarche communément adoptée rejoint ainsi la position défendue entre autres par É. Barjolle (2001) ou encore C. Vargas (2009) à la suite de B. Lahire (1993), pour qui il est didactiquement préférable de partir d'énoncés contextualisés, plutôt que de phrases isolées, le travail sur les formes gagnant en efficacité si le corpus d'observation fait sens pour l'enfant, lequel vit «dans l'univers concret des énoncés en situation » (Vargas, 2009, p. 28).

43 En y regardant d'un peu près toutefois, il s'avère que le texte de départ des manuels (et partant, l'ensemble de l'activité de découverte) fonctionne en fait comme un support assez artificiel, puisque les questions qui l'accompagnent portent chaque fois sur des constituants isolés, typographiquement mis en évidence (avec des questions sur «les mots en rouge ", "les groupes en gras »...), et la lecture de l'ensemble du texte n'est nullement nécessaire pour y répondre. En effet, l'unité même du texte support n'est jamais questionnée ${ }^{16}$, si bien que la phase de recherche initiale propose finalement une approche morcelée du texte, sans prise en compte de la contribution sémanticoréférentielle des expansions à l'interprétation du discours ${ }^{17}$.

Ainsi que nous l'avons signalé, deux manuels se démarquent des autres en ne recourant pas au texte lors de la mise en activité des élèves. C'est le cas du manuel 11, qui propose une succession de phrases décontextualisées immédiatement suivies de questions, et qui constitue paradoxalement l'un des ouvrages qui accorde le plus d'importance à la contribution sémantique des expansions. Le manuel 4, enfin, part d'une «situation problème ", qui s'avère finalement très guidée : un corpus de différents $\mathrm{GN}$, présentés 
comme des titres de livres et déjà classés selon le critère implicite du type d'expansion, est soumis à l'observation des élèves qui doivent justifier le classement proposé. À noter qu'en dépit de la présence d'un texte support, les autres manuels proposent souvent des activités similaires. Dans le manuel 5 par exemple (p. 199), il faut classer les groupes de mots en trois colonnes selon la couleur qui leur a été affectée dans le texte, puis donner un titre à chaque colonne.

\subsubsection{Quel questionnement de départ ?} censés illustrer.

En ce qui concerne la nature des constituants portés à l'attention des élèves dans le questionnaire qui accompagne le texte support, il peut s'agir selon les cas de l'ensemble du GN expansé, de l'expansion seule, ou encore du $\mathrm{N}$ seul. Charge ensuite à l'élève d'identifier le nom-tête ou les expansions.

Dans les cas où l'ensemble du GN est typographiquement mis en évidence, certains manuels se focalisent d'abord sur la structure syntaxique du groupe. C'est le cas du manuel 1 (p. 41) dont nous reproduisons un extrait à titre illustratif :

«[...] votre tête ressemble à un ballon de football » $\rightarrow 1$. Relève le nom principal dans le groupe nominal en couleur [ici en italiques]; 2. Quel mot ne peut-on pas supprimer ?; 3. Quelle est sa nature?

D'autres supports proposent une approche purement sémantique, comme le manuel 3 (p.46) dans lequel on note au passage le caractère artificiel de la mise en recherche puisque le rôle des expansions est donné dans la question :

«Ce sont de petits arbres un peu tordus avec de jolies fleurs roses qui sentent très bon.» $\rightarrow$

Souligne les mots ou groupes de mots qui donnent des informations sur les arbres.

À noter toutefois que les élèves sont parfois invités à formuler eux-mêmes le rôle sémantique des expansions, comme dans le manuel 10 (p.172) où la pertinence du corpus n'a toutefois rien d'évident :

«C'est à ces heures que les rayons ultraviolets (UV) émis par le soleil sont les plus intenses. [...] Pour vos activités de plein air, recherchez les endroits ombragés [...] Les lunettes de soleil sont indispensables [...]» $\rightarrow$ « 1 . Relève les groupes de mots en bleu [ ici en italiques] et entoure dans chacun d'eux le nom noyau. 2. A quoi servent les autres mots? 3. Peut-on les supprimer?»

En effet, le passage de « recherchez les endroits ombragés » à " recherchez les endroits » induit par la question 1 donne un résultat plutôt incongru (ce qui en soi est intéressant, mais ne correspond manifestement pas à l'objectif généralisant du manuel). Et la question 3, qui demande d'identifier le constituant facultatif du SN « les rayons ultraviolets » n'a rien d'évident non plus (la substantivation les ultraviolets étant courante). S'il est clair qu'une approche des faits de langue basée exclusivement sur des exemples canoniques n'est pas souhaitable pour la classe (en plus d'être étrangère à la réalité du discours, elle constitue un frein au développement d'une posture métalinguistique réflexive), on peut toutefois s'interroger sur la pertinence de certains exemples présentés comme prototypiques, alors même qu'ils sont précisément à la marge de la règle qu'ils sont

50 Dans certains manuels, seul le $\mathrm{N}$ est mis en évidence, et il s'agit pour les élèves de relever les expansions. La démarche est alors sensiblement différente : le cadre clos du GN n'étant pas donné d'emblée, la possibilité existe alors que certains élèves identifient comme expansion des éléments extérieurs au syntagme. Dans le manuel 6 (p. 50), par exemple, le 
raisonnement porte sur un énoncé attributif qui autorise, étant donné la consigne, à identifier l'attribut comme une expansion :

«Les traces de la carriole étaient nettement visibles sur le bas-côté.» $\rightarrow$ « Relève

les mots qui précisent le nom traces"

51 Un troisième cas de figure est fourni par les manuels qui isolent des expansions seules, les élèves devant alors retrouver le $\mathrm{N}$ modifié. D'un point de vue référentiel, ce choix peut toutefois sembler discutable dans la mesure où il est vraisemblablement plus complexe pour les élèves de raisonner à partir d'une propriété adjectivale abstraite plutôt que du support d'une référence nominale.

Signalons enfin que deux manuels proposent un texte sans aucun balisage, les élèves devant eux-mêmes relever les GN (cf. manuel 9 p. 46: «Observe les différents groupes nominaux de cet article de journal. Pour chacun, relève le nom noyau »). Si la démarche peut être intéressante, elle pose toutefois le problème du pré-requis, puisqu'elle implique la connaissance préalable des constituants du GN. Ainsi que le soulignaient déjà P. Péroz et M. Delaborde (2005, p. 182), «les tâches d'identification qui [...] sont généralement demandées en langue sont d'autant plus difficiles à accomplir qu'elles demandent que les apprenants possèdent déjà le modèle abstrait que la leçon est censée construire ».

En résumé, bien que les manuels examinés partent d'un texte support, ce dernier n'est jamais examiné en lui-même, et les dispositifs conçus pour la mise en activité des élèves proposent des questions très guidées portant chaque fois sur des constituants ponctuels, avec une réflexion sur le sens plutôt sommaire et un saut rapide vers la leçon. Si les questions invitent le plus souvent à recourir aux opérations traditionnelles de suppression et ajout de l'analyse distributionnelle, nous avons vu qu'elles n'impliquent pas nécessairement pour autant de véritables situations de recherche susceptibles de rendre les élèves réellement acteurs de leur apprentissage. Cela peut sembler dommageable dans la mesure où comme le rappelle C. Tisset (2005, p. 22, dans la lignée des travaux de J. Piaget, L. Vygotski, J. Bruner) les enfants apprennent mieux quand ils sont acteurs et chercheurs d'explication.

\subsection{La règle proposée}

54 La dernière partie de notre contribution portera sur l'analyse de la règle, telle qu'elle est proposée dans les manuels.

\subsubsection{Patron récurrent}

La leçon qui suit la phase de mise en activité s'articule le plus souvent en deux temps, avec d'abord un mot sur le rôle sémantique des expansions ("à quoi ça sert? »), puis des considérations syntaxiques avec une liste des constituants susceptibles de compléter le nom.

Du point de vue de la contribution au sens d'abord, on observe un recours massif aux notions d'enrichissement et de précision (cf. « enrichir le nom ", " donner des précisions sur le nom», "préciser le sens d'un nom», «apporter une information »... $)^{18}$. À noter qu'aucun manuel n'aborde la distinction entre les expansions explicatives et les expansions déterminatives ${ }^{19}$.

57 Syntaxiquement, la plupart des manuels attire l'attention des élèves sur le caractère facultatif des expansions, sans jamais discuter la propriété. Ceci peut toutefois sembler 
problématique dans la mesure où les exemples proposés ne se prêtent pas tous à l'effacement (cf. l'énoncé discuté supra : «? Pour vos activités de plein air, recherchez les endroits»). Concernant le caractère cumulable des expansions, seul le manuel 3 le mentionne explicitement (p. 46), et un coup d'œil aux exercices de manipulation proposés par les manuels montre qu'ils se basent le plus souvent sur des corpus forgés de GN à expansion unique, ne reflétant que très partiellement la réalité de l'usage en discours. Les propriétés syntaxiques des expansions sont donc relativement peu mises en avant, alors même que les opérations de l'analyse distributionnelle mobilisées dans la phase de questionnement initial constituaient une occasion de les mettre en lumière.

Enfin, si 8 manuels présentent les expansions comme des moyens de compléter le nom seul, 5 autres $\left(n^{\circ} 1,2,6,7,11\right)$ les présentent comme des compléments du GN dans son entier ${ }^{20}$.

\subsubsection{Le mélange des plans et le risque de confusion « nature/fonction »}

En ce qui concerne la liste des constituants susceptibles de modifier le nom, il est frappant de constater que la plupart des manuels consultés propose des listes mêlant indifféremment natures et fonctions, la tendance la plus fréquente consistant à mettre la fonction de "complément du nom » sur le même plan que les catégories grammaticales de l'adjectif et de la relative ${ }^{21}$. Cette "confusion des plans» est sensible dès les sommaires: les titres de chapitres recensés supra (section 2.2.1) font clairement état d'une telle approche, qui caractérise 8 des 12 manuels examinés. La rubrique « Autour du nom» du manuel 2 consacre ainsi un chapitre à «l'adjectif qualificatif», un autre au « complément du nom », et un à « la proposition subordonnée relative». Le manuel 6 va même plus loin en coordonnant explicitement « le complément du nom et la proposition relative ".

Dans le corps même du chapitre dédié aux expansions, plus de la moitié des manuels mentionne le "complément du nom» à la suite de l'adjectif qualificatif et de la proposition relative, délaissant la notion de groupe prépositionnel pourtant attendue dans un tel contexte. Le manuel 1 (p. 42) propose ainsi la synthèse suivante :

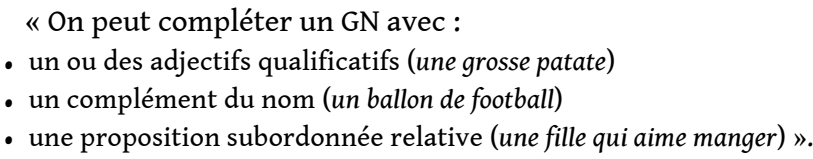

61 Le risque de confusion est encore accru dans le manuel 11. On y lit (p. 42) :

"Les expansions du groupe nominal [en orange dans le manuel] peuvent être :

des adjectifs qualificatifs qui précisent le nom. Ils sont épithètes du nom: un perroquet vert; ou ils sont apposés au nom : joyeux, le perroquet siffle.

des compléments du nom qui complètent le nom. Ils peuvent être :

- un groupe nominal : une tasse à café

- un verbe : un piano à vendre

une proposition relative : un meuble qui sert à ranger des disques ".

62 Les choix typographiques et la formulation d'une telle synthèse mettent finalement en regard deux listes "mixtes »: la liste "adjectif, complément du nom, relative » d'une part; et la liste "épithète, apposé, GN, verbe » d'autre part. On observe également qu'aucune mention n'est faite de la préposition dans le cas du complément du nom.

Outre le fait qu'elle soit assimilée à une nature, on peut aussi noter que l'étiquette de « complément du nom » est utilisée dans un sens restreint, puisqu'elle est le plus souvent 
réservée aux seuls groupes prépositionnels, alors que comme le rappellent $\mathrm{M}$. Riegel et al. (1994, p. 179), tous les modificateurs du $\mathrm{N}$ fonctionnent comme de véritables compléments ${ }^{22}$.

64 Enfin, il arrive aussi que la notion d'expansion soit mentionnée dans un aide-mémoire général sur les fonctions, parfois placé en fin d'ouvrage, mais ce qui se présente alors comme un « mémo » offre rarement une vision synthétique du système. Outre le fait que les listes proposées mêlent parfois indifféremment les compléments essentiels du verbe et les compléments facultatifs de la phrase et/ou du nom, les fonctions sont rarement appréhendées en terme relationnels, ce qui n'œuvre pas en faveur d'une conscience claire du rôle des mots, ni de la distinction nature $v$ s fonction.

\subsubsection{La part de l'implicite}

Soulignons pour terminer que la présence de telles listes fourre-tout est rendue possible par le fait que la distinction syntaxique «nature/fonction" n'est presque jamais explicitement posée. L'implicite occupe ainsi une bonne place dans les leçons des manuels. Seuls deux d'entre eux ${ }^{23}$ recourent explicitement au métalangage de nature et de fonction. Dans les autres cas, le fait que le terme d'expansion renvoie à une fonction, potentiellement assumée par différentes classes de constituants n'est jamais explicitement précisé. Charge alors à l'élève de comprendre de quoi sont faites les listes proposées dans les leçons, et de situer la notion générale d'expansion dans le paysage complexe de l'analyse grammaticale. Un manuel du corpus ${ }^{24}$ réserve d'ailleurs le terme syntaxique de "fonction » aux seuls compléments du verbe, les expansions du nom étant quant à elles étiquetées sémantiquement comme des "enrichissements ». Ce recours frileux au métalangage peut pourtant sembler préjudiciable à l'élève qui ne dispose pas alors de termes précis pour bâtir un raisonnement grammatical, et se retrouve condamné à une approche ponctuelle de faits particuliers sans possibilité d'accéder à une vue d'ensemble du système, qui lui permettrait par exemple de mettre en regard les compléments du verbe, de la phrase et du nom. Ainsi que l'ont montré M. Nadeau et C. Fisher (2014, p. 176), le fait d'éviter l'emploi du métalangage grammatical en classe est en outre d'autant plus dommageable que cela prive les élèves de « la possibilité d'accéder à un niveau de généralité (d'abstraction) dont ils ont besoin pour transférer leurs connaissances en écriture ».

Notons pour finir que la part de l'implicite dans les manuels vient également de ce que plusieurs d'entre eux pratiquent en fait une grammaire par l'exemple, notamment avec les SN binominaux du type "la tour Eiffel », à propos desquels il est simplement précisé que le complément du nom est parfois placé juste après le nom, sans préposition. Aucune autre explication n'accompagne les exemples, ce qui revient à pratiquer une grammaire implicite, à partir de constructions dont l'analyse fait justement débat en littérature. Si on considère un exemple comme "la tour Eiffel», on sait bien en effet que certaines grammaires parlent de «noms épithètes " (cf. Riegel et al., 1994), là où d'autres parlent " d'appositions liées », ou encore de " complément déterminatif » (cf. Grevisse, 1988). On peut aussi se poser la question de savoir si tous les exemples proposés relèvent d'une analyse unitaire (cf. «danseuse étoile», qui évoque la composition, à côté de "tour Eiffel »). Outre le fait que la grammaire implicite soit le plus souvent préjudiciable à l'apprenant, la structuration des acquis ne pouvant passer que par un discours explicite (cf. notamment Vigner, 1984; Tisset, 2005 ; Damar, 2009, ou encore Fisher \& Nadeau, 
2014), la question semble ici d'autant plus légitime que les exemples donnés aux élèves ne font pas consensus dans la littérature.

\section{Bilan}

L'objectif initial de ce travail était de sonder les représentations et compétences des élèves autour de la notion d'expansion du nom, et de dresser un état des lieux du traitement de la notion dans les manuels scolaires.

Du côté des élèves, les réponses qu'ils proposent se basent souvent sur des intuitions pertinentes, mais très peu d'entre eux semblent avoir une vision unifiée du rôle des mots dans la phrase. La confusion entre les différents plans d'analyse (en particulier la distinction nature/fonction) est fréquente et le recours maladroit au métalangage suggère qu'il ne fait pas sens pour les élèves. Précisons aussi que ces grandes tendances valent pour le public interrogé, sans que la taille restreinte de notre sondage ne nous permette de tirer de conclusions définitives quant au public scolaire.

Du côté des manuels, nous avons vu qu'en dépit d'une entrée par le texte, les expansions sont rarement appréhendées du point de vue de leur contribution au discours en lecture/ écriture, et que la phase de mise en activité des élèves s'avère rarement inductive. Un autre constat vient de ce que le recours au métalangage y est plutôt frileux, et que les différents plans de l'analyse grammaticale sont rarement mis explicitement en regard, si bien qu'au bout du compte, l'hypothèse d'une corrélation entre les représentations des élèves et le traitement des expansions dans les manuels semble tout à fait probable. Le peu d'attention porté au caractère généralement facultatif des expansions, par exemple, ne permet pas de les distinguer des compléments du verbe, qui à leur façon «enrichissent» aussi le constituant qu'ils complètent, ni de les rapprocher des compléments de phrase, eux-mêmes facultatifs.

70 Pour autant, il va de soi que les manuels restent des supports susceptibles d'exploitation intéressante, dès lors que l'enseignant se les approprie personnellement. Cette prise en considération du pôle enseignant et des pratiques sur le terrain constitue d'ailleurs le prolongement à venir de notre recherche.

\section{BIBLIOGRAPHIE}

\section{Articles et ouvrages}

BARJOLLE, É. (2001). « Une gêne pratique à l'égard des manuels ou la phrase en mal de discours ». Le français aujourd'hui 135, p. 32-43. BENTOLILA, A. (2006). Rapport de mission sur l'enseignement de la grammaire. En ligne : http:// media.education.gouv.fr/file/68/3/3683.pdf. 
DAMAR, M.-E. (2009). Pour une linguistique applicable. L'exemple du subjonctif en FLE. Bruxelles et al. : Peter Lang.

DELHAY, C. (2014). « Pour un complément attribut ». Repères 49, p. 57-76.

FISHER, C. \& NADEAU, M. (2014). « Usage du métalangage et des manipulations syntaxiques au cours de dictées innovantes dans des classes du primaire ». Repères 49, p. 169-171.

GARDES-TAMINE, J. (2005). De la phrase au texte. Enseigner la grammaire du collège au lycée. Paris : Delagrave Édition.

GREVISSE, M. (1988). Le Bon Usage. Grammaire française, $12^{\mathrm{e}}$ éd. refondue par A. Goes (2 $2^{\mathrm{e}}$ tirage). Paris-Gembloux : Duculot.

LAHIRE, B. (1993). Culture écrite et inégalités scolaires. Lyon : Presses universitaires de Lyon.

PELLAT, J.-C. (2009). Quelle grammaire enseigner? Paris : Hatier.

PÉROZ, P. \& DELABORDE, M. (2005). « Un exemple de manipulation grammaticale : le tri de phrases au CE2 ». Pratiques 125-126, p. 179-187.

RIEGEL, M., PELLAT, J.-C., RIOUL, R. (1994). Grammaire Méthodique du français. Paris : Presses universitaires de France.

TISSET, C. (2005). Observer, manipuler, enseigner la langue au cycle 3. Paris : Hachette.

VARGAS, C. (2004). « La création des savoirs à enseigner en grammaire : de la recomposition à la reconfiguration ». In : Vargas, C. (dir.), Langue et études de la langue. Approches linguistiques et théoriques. Aix-en-Provence: Presses universitaires de Provence, p. 35-48.

- (2009). «Peut-on inventer une grammaire pour la réussite scolaire ? » Repères 39, p. 17-39.

VIGNER, G. (1984). L'exercice dans la classe de français. Paris : Hachette.

\section{Documents institutionnels}

Programmes de l'école primaire, BO hors-série nº 3 du 19 juin 2008.

Programmes d'enseignement des cycles 2, 3 et 4, BO spécial nº 10 du 19 novembre 2015.

\section{Manuels Scolaires}

ADAD, D., LORMEL, E., MAINVILle, T. \& VAUtRot, A. (2011). Étude de la langue. Paris : Bordas.

ANNARUMMA, S., RAIMBERT, S., VANETTI, C. \& VARIER, M. (2010). Français livre unique. Paris : Istra.

BARTHOMEUF, C. (2013). Les nouveaux outils pour le français, CM2. Paris : Magnard.

Benaych, P., CRinon, J., GALlet, D., GUÉRAult, D., LALlias, JC., PACHet, S. \& SEBAG, S. (2009). L'île aux mots. Paris : Nathan.

BONDOt, A., GONNET, Y., GONNET, M., HOSTEAU, G., PICOT, F. \& PIGNON, M. L. (2013). Mille-feuilles, CM2. Paris : Nathan.

BRAILleT-PASQUEREAU, P., CASTERA, C. \& VERKINDRE-RIPARD, I. (2010). Interlignes. Étude de la langue. Les Mureaux : Éditions Sed.

CAmus, C., Da silva, M., foussard, E., mainville, O., VAutrot, A. \& GRumel, O. (2013). Mots en herbe. Paris : Bordas. 
CAUTElA, A., LE GUAY, I., MARIN, B., ROBERT, N. \& ROBINET, F. (2012). Étude de la langue, Cycle 3. Paris : Nathan.

CAYlat, M., Degoul, L., MAURouARD, M. A., MousSAoui, S. \& SAVAdouX-WojCiechowski, C. (2013).

Pépites Français CM2. Paris : Magnard.

CHUARD-HENDRIKS, DELAPORTE-EL ADRHAM, A. \& SCHROEDER, Y. (2012). Mon livre de français CM2. Les

Mureaux : Éditions Sed.

DE RAM, C., KNOWLES, X., LEMAIRE, M. \& PARÉ, S. (2011). Mot de Passe. Paris : Hachette Éducation.

SCHÖTTKE, M. \& TOURNAIRE, F. (2011). Français CM1-CM2. Étude de la langue. Paris : Hatier.

\section{ANNEXES}

\section{Annexe : Questionnaire soumis aux élèves ${ }^{25}$}

I) En grammaire, qu'est-ce qu'une « expansion du nom » ? Essaie d'expliquer le plus clairement possible, en précisant à quoi ça sert. Tu peux ensuite donner des exemples pour illustrer les différents types d'expansions du nom que tu connais.

\section{II) Précise la nature et la fonction des mots en gras}

1. Cette pièce est remplie d'objets anciens et de meubles qui sentent la cire. 2 . La danseuse porte une magnifique robe à paillettes. 3. Jules a acheté un ordinateur d'un prix excessif. 4. Cet homme a commis un crime que l'on ne peut pas accepter.

III) Recopie les phrases en rajoutant une expansion au nom en gras. Essaie d'utiliser à chaque fois un type d'expansion différent!

1. Mes parents ont acheté un chien. 2. Cette femme ressemble beaucoup à son père. 3 . Mes voisins vendent leur maison.

\section{IV) Entoure les expansions des noms en gras}

1. La cueillette des fraises est un travail épuisant pour les ouvriers. 2. Ce matin, un énorme rocher est tombé dans la mer du haut de la falaise. 3. La robe qui se trouve dans cette vitrine me fait vraiment très envie. 4 . Le frère de mon voisin est malade. 5 . Il a une grande sœur qui fait des études à Paris.

\section{NOTES}

1. Conformément à l'usage communément en vigueur à l'école (dans les manuels notamment). On précisera toutefois que cette terminologie d'« expansion du nom », qui a figuré un temps dans les programmes scolaires, ne figure ni dans les programmes pour le primaire de 2008, ni dans les programmes pour la rentrée 2016.

2. Cf. aussi, dans une perspective directement didactique, l'ouvrage de J.-C. Pellat (2004).

3. Nous remercions A. Corbillon et E. Deledalle d'avoir soumis ce questionnaire à leurs élèves. Le sondage a été réalisé en octobre 2014, au collège Saint Paul (59).

4. C'est nous qui mettons en gras. 
5. Cette tendance, qui peut s'expliquer par l'homonymie entre que pronom relatif et la conjonction, suggère qu'il aurait été intéressant d'intégrer dans le corpus une subordonnée initiée par un autre relatif, afin de voir si la forme du pronom impacte ou non les réponses des élèves.

6. Cf. en annexe corpus de l'activité 4 .

7. L'expérience du terrain nous a aussi appris que la difficulté des élèves à concevoir la relative comme un modifieur du nom s'explique également par la présence d'un verbe conjugué, perçue comme « anormale » dans le cadre du GN.

8. Dans l'activité 2 , par exemple, un quart des réponses propose une classe grammaticale là où il s'agissait d'indiquer une fonction, et on retrouve $20 \%$ de fonctions dans les natures.

9. Les références complètes des manuels, avec mention des noms d'auteurs, figurent dans la bibliographie en fin d'article.

10. Le chiffre qui précède le titre renvoie à la place du chapitre dans le sommaire, même si la numérotation n'est pas toujours explicite dans les manuels du corpus.

11. Cette préconisation figure dans les programmes pour 2016 (cf. «Terminologie utilisée » au cycle 3, Bo du 26 novembre 2015, p.118).

12. Dans le manuel 11 par exemple, les pronoms personnels sont abordés dans la rubrique dédiée au verbe, entre les compléments circonstanciels et l'attribut du sujet. Le même manuel utilise en outre la formulation "Autour de... » pour les rubriques de la partie vocabulaire (cf. les intitulés " Autour de la formation des mots », «Autour du sens »), ce qui exclut l'idée que " autour de... » renvoie aux fonctions par rapport à une catégorie syntaxique.

13. À noter que le manuel 7 parle d'expansion « du GN » (et non du N seul), sans toutefois traiter des expansions détachées de l'ensemble du GN.

14. Nous reviendrons infra sur la terminologie employée.

15. Sauf dans les manuels 6 et 8 qui abordent ensemble « le complément du nom et la relative ».

16. À l'exception du manuel 12, qui initie une réflexion à partir de « la recette du cake salé » (p. $150)$, envisagée dans son ensemble en tant que discours prescriptif.

17. Ce constat d'une induction « de surface » n'est pas nouveau : il rejoint par exemple celui d'É. Barjolle (2001) dont l'analyse portait sur le traitement des types de phrases dans un corpus de manuels de 1996 à 2000.

18. Les manuels 1 et 6 se cantonnent toutefois au versant syntaxique, les expansions étant présentées comme ce qui permet de « compléter le groupe nominal ».

19. Le manuel 12 précise cependant: «Généralement les expansions du nom sont facultatives. Cependant, dans certains cas elles sont essentielles : L'étoile de mer appartient au milieu marin » (p. 150).

20. Le manuel 11 est toutefois le seul à mentionner l'adjectif en position détachée.

21. Cette tendance fait vraisemblablement écho à la formulation du BO de 2008, qui évoque la nature possible $\mathrm{du}$ «complément $\mathrm{du}$ nom » dans le seul cas de la relative, sans mentionner le groupe prépositionnel (cf. «l'identification des éléments du groupe nominal et de leurs fonctions : déterminant, adjectif qualificatif épithète, complément du nom, proposition relative complément du nom »).

22. Seuls 5 manuels du corpus étendent la notion de complément à la relative. On note aussi qu'aucun manuel ne fait mention des complétives complément du nom.

23. Il s'agit des manuels 4 et 12 .

24. Cf. mémo du manuel 2, p. 160-162.

25. Ce questionnaire visait bien à sonder un état de connaissances et de compétences : il ne constitue en aucun cas un dispositif didactique d'enseignement/apprentissage pour la classe. 


\section{RÉSUMÉS}

Ce travail porte sur les expansions du nom envisagées selon une perspective didactique, l'objectif étant de sonder les représentations et compétences des élèves, pour pouvoir ensuite les confronter au traitement de la notion dans les manuels scolaires. La première partie de la contribution présente ainsi les résultats d'une enquête de terrain, menée auprès d'un groupe d'élèves de début de collège. La seconde partie dresse un état des lieux du traitement des expansions dans la littérature scolaire, à partir de l'examen de 12 manuels de CM2 [dernière année de l'école primaire]. L'analyse porte sur les dispositifs didactiques mis en place pour aborder la notion, mais aussi sur la formulation de la leçon proposée, ce qui permet au passage de mesurer l'écart éventuel entre le discours scolaire et les grammaires de référence.

The present research focuses on noun modification from a pedagogical perspective. The goal is to investigate what pupils know and are capable of doing with respect to noun modification, and to compare this with the treatment of noun modification in textbooks. We first present the results of a field study carried out on students at the beginning of middle school (around 11 years of age). We then describe how noun modification is dealt with in textbooks through an examination of 12 textbooks designed for pupils in their final year of primary school. In the discussion, we consider the pedagogical strategies used to tackle the topic of noun modification, as well as how the proposed lesson is constructed. Finally, this approach allows us to examine the potential gap between educational discourse as found in textbooks and the language used in reference grammars.

INDEX

Keywords : noun modification, grammar teaching, textbooks, syntactic category, syntactic function

Mots-clés : expansions du nom, didactique de la grammaire, manuels scolaires, représentations d'élèves, catégorie syntaxique, fonction syntaxique

\section{AUTEUR}

\section{CÉLINE CORTEEL}

